\title{
Evolution of Modern Recommender Systems with AI Techniques
}

\author{
Artyom Kravchenko
}

\begin{abstract}
Recommender systems (RSs) emerged several decades ago as software tools analyzing Web user preferences and making intelligent product and content suggestions based on their behavior and tastes. At first, they utilized simple comparison algorithms and analysis of explicitly stated user data in the form of ratings and likes. With the booming expansion of Web data and growing variety of products, services, and information sources online, RSs face pressing challenges to efficiency of recommendations such as data sparsity, cold start, and scalability requirements. The solutions to these issues come in the form of AI-based techniques - advanced machine learning algorithms that adapt to the Web landscape and address the user requirements flexibly. With the trend of Web expansion certain to remain unchanged for many years to come, intelligent, AI-enriched RSs are expected to gain greater significance in users' decision-making and Web navigation. But to gain user trust and ensure a greater user buy-in, RSs have to offer transparency and perceived credibility.
\end{abstract}

\section{Introduction}

Today Internet users have to navigate in the endless (and expanding) amounts of information and product variety, which are incomprehensible for a single individual. Thus, the problem of relevant resources' findability has arisen in the technology sector, and its solution came in the form of recommender systems (RSs) (Manouselis, Drachsler, Vuorikari, Hummel, \& Koper, 2012). These systems are an integral part of intelligent mechanisms for sorting out the Internet content and making suggestions for users based on their tastes and preferences. Such recommendations are traditionally provided by smart search engines and web marketing resources based on the users' previously viewed content, collaboration (likes and ratings provided by the user), and demographics (Azam \& Yao, 2014). The suggestions that recommender software tools provide to users relate to a variety of decision-making processes, such as items for purchase, video and audio content to consume, news feeds to read, and tourist destinations to attend.

With user guidance in a personalized way to interesting objects and information in the large space of Internet, RSs have proven efficient in matching up the attributes of a user profile with the attributes of the content object. For instance, 2/3 of movies watched on Netflix are recommended movies, while $38 \%$ of click-through rates on Google News and 35\% of sales at Amazon also come from the system's recommendations (Gorakala, 2016). Thus, 
investment in cutting-edge, flexible, advanced RSs is imperative for business growth today as an ability to provide genuinely relevant recommendations is an effective sales driver.

\section{How RSs Work}

RSs, (also referred to as advice-giving systems, recommendation engines, and recommendation agents) are defined as "a branch of information retrieval and artificial intelligence" and as "powerful tools and techniques to analyze huge volumes of data, especially product information and user information, and then provide relevant suggestions based on data-mining approaches" (Gorakala, 2016, p. 7). RSs come to Web users' rescue when it comes to provision of relevant, required information corresponding to the user's search query. They act on the basis of mathematical models and objective functions predicting how much a user may like a certain suggested item.

Modern RSs focus on specific types of items such as music, news, or tourist destinations, and their graphical user interface (GUI), design, and the core recommendation technique utilized for the generation of suggestions are all specifically tailored to generating effective, relevant recommendations on them (Ricci, Rokach, \& Shapira, 2010). The purpose of RSs is to advise content or items to users lacking a personal experience in some area for making a relevant, useful purchase or choice of free content. For instance, Amazon employs AI-powered algorithms to customize the look and feel of its e-store for each individual visitor and deliver a personalized shopping and reading experience (Smith \& Linden, 2017).

Any web resource's RS is designed in such a way that analyzes each individual user's digital footprint (e.g., socio-demographic information, transaction details, interaction logs) and compares the derived data with the particular product's information (e.g., its features,

feedback from users, comparison with other products). Sources of such data include explicitly stated information in the form of ratings and likes given to some products and implicit data derived from the user's behavior on the website and the search engine history derived from cookies on the user's device. By collecting and analyzing this data, the RS can predict whether particular users may prefer an item or not, thus simplifying their decision making in the complex information environments (Isinkaye, Folajimi, \& Ojokosh, 2015).

Creating a good, effective recommendation engine is a task of strategic importance for both consumers and vendors. On the customer's side, a good recommendation system guarantees receiving relevant suggestions and quicker, better decision making, which leads to 
consumer confidence and loyalty. On the seller's side, deep analysis of consumer data and custom-tailored product suggestions promise exponential sales increases. Thus, the evolution of RSs takes place together with the expansion and growing sophistication of online systems, with challenges of recommendations in real time, big-data analysis, and AI integration to be met for the sake of focused, personalized user service (Gorakala, 2016).

\section{How RSs Evolved}

The first RS algorithms employed simple neighborhood methods and provided batchmode recommendations derived from similarity calculations. They analyzed only user ratings by means of calculating Euclidian distances, the Pearson coefficient, cosine similarity, etc. (Gorakala, 2016). They also followed the collaborative filtering rationale, which presupposed the provision of recommendations based on what other users with similar tastes and choices made. This approach suggested that since the user agreed with other users in their choices and ratings before, their later choices would also be appealing to him or her (Arazy, Kumar, \& Shapira, 2009).

However, the principle failed to meet the demand for efficient and customized recommendations in the e-commerce area, with e-shops possessing huge assortments of items and requiring RSs that would give sensitive, smart suggestions based on the whole range of available alternative products (Falk, 2019). In addition, the overall global expansion of Web data and product offerings has led to the decrease of users' well-being and inability to make wise choices amid the information overload. Smart RSs based on the AI advancement, machine learning, and the principles of user-adapted machine interaction emerged to address this phenomenon and enable users to navigate in the abundant Web data.

It is notable that RSs operating on the simple user-based and item-based collaborative filtering approaches are still in place and work quite well. The former generates recommendations based on the analysis of the user's neighborhood and similar user preferences, while the latter finds similarities between items and recommends non-rated items similar to those rated by the user in the past. But along with the technological progress, RSs also evolved into personalized, context-aware recommenders providing suggestions in real time based on accurate, complex machine-learning approaches. Many of them apply advanced computational models such as matrix factorization and singular value decomposition to address the changing needs of users and embrace exponential Web data growth (Gorakala, 
2016). Hybrid RSs are increasingly adopted as more robust recommender solutions because of combined strengths of user-based and item-based systems. They issue more accurate suggestions by analyzing aggregate data on the user and the item, thus leveraging the power of multiple data sources and improve the performance of existing RSs with particular data modalities (Aggarwal, 2016).

\section{AI-Enriched RSs}

Modern RSs have to become much more than they used to be even a decade ago as the pressure of the increasing information overload in the Web is a serious challenge to their efficiency. An efficient RS design has to address the problems of scalability, sparsity, and cold start, as well as the need for ubiquitous information processing in the Web (Gabrani, Sabharwal, \& Singh, 2016). Such needs led to the development of AI-based, context-aware, intelligent RSs capable of managing the information overload and filtering. Contemporary RS progress is in part attributable to the use of advanced artificial intelligence (AI) techniques improving recommendation accuracy and sustainably mitigating the aforementioned issues. Such techniques presently used in RSs include fuzzy sets, artificial neural networks, evolutionary computing, swarm intelligence, and artificial immune systems (Abbas, Zhang, \& Khan, 2015).

One of the most common AI technologies applied in RSs is collaborative filtering; the challenge of its application was dealing with data sparsity at the cold start, which means having little rating information from the user to base suggestions on. The solution to this problem was found in the involvement of transfer learning techniques by leveraging a rich collaborative body of knowledge from other similar systems running for a long time. Zhao, Pan, and Yang (2017) suggested the use of maximum-margin matrix factorization, regularized low-rank matrix factorization, and probabilistic matrix factorization to maximize the utility and output of cross-system knowledge transfer and improve recommendation quality.

AI-based digital assistants now become available in large numbers and for a variety of purposes. Many tech giants make use of AI-based recommenders and assistants today (e.g., Microsoft, IBM, Google, and Amazon). Some recent examples include the chatbots incorporated into the Facebook Messenger system or Amazon Alexa, a digital recommender of the marketplace (Maedhe et al., 2019). eBay and Netflix are also known to use AI recommender techniques to provide personalized suggestions and customize product/service 
offerings to individual clients. In addition, the machine-learning library Mahout developed by Hadoop provides a flexible infrastructure for building, evaluating, and streamlining a variety of RS algorithms and makes RSs scalable (Verma, Patel, \& Patel, 2015). Another AI-powered RS technology is Apache Spark Streaming - a solution enabling scalability of big data and generating recommendations in real time (Meng et al., 2016). Neo4j is also a popular innovative tool for design of real-time graph-based RSs enabling fast retrieval and searching (Pellegrino, 2017).

\section{AI Application in RSs: Future Prospects}

The Web grows at an enormous pace, and billions of Internet users find it increasingly hard to make decisions and find relevant information in such a multitude of data. Thus, the future of AI-based RSs is optimistic given their intelligent ability to process large data volumes, interact seamlessly with Web users, learn by experience, and develop more sophisticated suggestion algorithms based on the analysis of user data. However, Bigras et al. (2018) pointed out the need for AI-based recommendation agents to offer richness of information and a variety of suggestions to end users for the sake of gaining credibility and being adopted. Therefore, while RSs continue to evolve to improve the users' decisionmaking processes, they need to be developed with the user's adoption intention in mind, which is influenced by the perceived decision quality.

Zhao, Benbaast, and Cavusoglu (2019) also pointed out the need to ensure transparency in RSs because its lack is what impairs users' adoption of RSs and hinders the efforts to assist clients in decision-making and maximize business profits. Unlike RSs of the past that explicitly asked the users to indicate their preferences and needs, modern AI-based RSs collect data and analyze it implicitly via smart collaborative filtering an content-based filtering techniques, which arouses privacy concerns among users and causes confusion about how a certain recommendation was generated. Transparency thus determines the extent to which information of a system's reasoning is provided and made available to users, and the provision of adequate explanations of is seen as the underlying factor of greater user buy-in of RSs.

\section{References}

Abbas, A., Zhang, L., \& Khan, S. U. (2015). A survey on context-aware recommender systems based on computational intelligence techniques. Computing, 97(7), 667-690. 
Aggarwal, C. C. (2016). Recommender systems: the textbook. New York, NY: Springer.

Arazy, O., Kumar, N., \& Shapira, B. (2009). Improving social recommender systems. IT Professional, 11(4), 38-44.

Azam, N., \& Yao, J.T. (2014). Application of game-theoretic rough sets in recommender systems. In M. N. Murty, et al. (Eds.), Multi-disciplinary trends in artificial intelligence: 8th international workshop, MIWAI 2014. Brussels, Switzerland: Springer International Publishing.

Bigras, E., Jutras, M.-A., Senecal, S., Leger, P.-M., Black, C., Robitaille, N.,... \& Hudon, C. (2018). In AI we trust: characteristics influencing assortment planners' perceptions of AI based recommendation agents. International Conference on HCI in Business, Government, and Organizations 2018, 3-16.

Falk, K. (2019). Practical recommender systems. New York, NY: Manning Publications Company.

Gabrani, G., Sabharwal, S., \& Singh, V. K. (2017). Artificial intelligence based recommender systems: a survey. International Conference of Advances in Computing and Data Sciences 2016, 50-59.

Gorakala, S. K. (2016). Building recommender systems. Birmingham, the UK: Packt Publishing Ltd.

Isinkaye, F. O., Folajimi, Y. O., \& Ojokoh, B. A. (2015). Recommendation systems: principles, methods and evaluation. Egyptian Informatics Journal, 16(3), 261-273.

Maedche, A., Legner, C., Benlian, A., Berger, B., Gimpel, H., Hess, T., ... \& Soellner, M. (2019). AI-based digital assistants. Business \& Information Systems Engineering, 110 .

Manouselis, N., Drachsler, H., Vuorikari, R., Hummel, H., \& Koper, R. (2012). Recommender systems in technology enhanced learning. In F. Ricci, L. Rokach, B. Shapira, \& P. Kantor (eds.), Recommender systems handbook. (pp. 387-415). Boston, MA: Springer.

Meng, X., Bradley, J., Sparks, E., Ventakataraman, S., Lui, D., .. \& Talwalkar, A. (2016). MLlib: machine learning in Apache Spark. Journal of Machine Learning Research, $17,1-7$.

Pellegrino, J. (2017). Flexible recommender systems based on graphs. AISR2017, Paris, France.

Ricci, F., Rokach, L., \& Shapira, B. (2011). Introduction to recommender systems handbook. In F. Ricci, L. Rokach, B. Shapira, \& P. Kantor (Eds.), Recommender systems handbook. (pp. 1-35). Boston, MA: Springer.

Smith, B., \& Linden, G. (2017). Two decades of recommender systems at Amazon.com. IEEE Internet Computing, 21(3).

Verma, J. P., Patel, B., \& Patel, A. (2015). Big data analysis: recommendation system with Hadoop framework. IEEE International Conference on Computational Intelligence \& Communication Technology, 92-97. 
Zhao, L., Pan, S. J., \& Yang, Q. (2017). A unified framework of active transfer learning for cross-system recommendation. Artificial Intelligence, 245, 38-55.

Zhao, R., Benbaast, I., \& Cavusoglu, H. (2019). Transparency in advice-giving systems: a framework and a research model for transparency provision. IUI Workshops 2019, Los Angeles, the USA. 